\title{
Giant monopole energies from a constrained relativistic mean-field approach
}

\author{
Wei-Chia Chen, ${ }^{1, *}$ J. Piekarewicz, ${ }^{1, \dagger}$ and M. Centelles ${ }^{2, \ddagger}$ \\ ${ }^{1}$ Department of Physics, Florida State University, Tallahassee, Florida 32306, USA \\ ${ }^{2}$ Departament d'Estructura i Constituents de la Matèria and Institut de Ciències del Cosmos, Facultat de Física, Universitat de Barcelona, \\ Diagonal 645, 08028 Barcelona, Spain
}

(Received 9 April 2013; revised manuscript received 8 July 2013; published 21 August 2013)

\begin{abstract}
Background: Average energies of nuclear collective modes may be efficiently and accurately computed using a nonrelativistic constrained approach without reliance on a random phase approximation (RPA).

Purpose: To extend the constrained approach to the relativistic domain and to establish its impact on the calibration of energy density functionals.

Methods: Relativistic RPA calculations of the giant monopole resonance (GMR) are compared against the predictions of the corresponding constrained approach using two accurately calibrated energy density functionals. Results: We find excellent agreement—at the $2 \%$ level or better-between the predictions of the relativistic RPA and the corresponding constrained approach for magic (or semimagic) nuclei ranging from ${ }^{16} \mathrm{O}$ to ${ }^{208} \mathrm{~Pb}$.

Conclusions: An efficient and accurate method is proposed for incorporating nuclear collective excitations into the calibration of future energy density functionals.
\end{abstract}

DOI: 10.1103/PhysRevC.88.024319

PACS number(s): 24.30.Cz, 21.60.Jz, 21.65.Cd, 21.65.Mn

\section{INTRODUCTION}

A tractable microscopic theory that both predicts and provides well-quantified theoretical uncertainties for nuclear properties throughout the nuclear chart is the guiding principle in the quest of a universal nuclear energy density functional [1,2]. Density functional theory (DFT) provides a powerfuland perhaps unique-framework for the accurate calculation of the ground-state properties and collective excitations of medium-to-heavy nuclei. Based on the seminal work by Kohn and collaborators [3-5], DFT shifts the focus from the complicated many-body wave function to the much simpler one-body density. By doing so, the formidable challenge of deducing the exact ground-state energy and one-body density from the many-body wave function "reduces" to the minimization of a suitable functional of the density. Of course, this enormous simplification comes at a price. Whereas DFT establishes the existence of a well-defined density functional, it offers no guidance on how to build it. Nevertheless, by strictly focusing on measurable quantities, DFT offers the opportunity for using physical intuition and symmetries/constraints to guide the construction of the functional. Moreover, Kohn and Sham have shown how the ground-state energy and corresponding one-body density may be obtained from a variational problem that reduces to a self-consistent solution of a set of mean-field-like ("Kohn-Sham") equations [4]. However, although the form of the Kohn-Sham equations is reminiscent of the self-consistent Hartree (or Hartree-Fock) problem in the presence of an underlying (bare) nucleonnucleon $(N N)$ interaction, the constants that parametrize the Kohn-Sham potential are directly fitted to many-body properties (such as masses and charge radii) rather than

\footnotetext{
*wc09c@my.fsu.edu

†jpiekarewicz@fsu.edu

${ }^{\ddagger}$ mariocentelles@ub.edu
}

two-body data. In this manner the complicated many-body dynamics gets implicitly encoded in the parameters of the model. In principle, a proper implementation of DFT and the Kohn-Sham equations incorporates all many-body effects in quantities that are functionals of the one-body density, such as the total ground-state energy [5].

The construction of an energy density functional (EDF) starts with the selection of terms that incorporate important symmetries and features of the nuclear dynamics. Although in principle such terms may be inspired by the underlying nucleon-nucleon dynamics, the explicit value of the coefficients in front of these terms (i.e., the model parameters) is customarily obtained through the minimization of a quality (e.g., $\chi^{2}$ ) function. Thus, the model parameters may-and in general do-differ significantly from the "bare" $N N$ values. This is a reflection of the fact that the parameters of the model are calibrated to physical observables that incorporate fewand many-body correlations. In the language of DFT, most of the complicated many-body dynamics gets implicitly encoded in the parameters of the model. Once the EDF has been properly defined, a set of accurately-measured ground-state observables is selected to constrain the model parameters through the minimization of a quality function. However, given that ground-state observables are fairly insensitive to fluctuations around the equilibrium density, one must often resort to "pseudodata"-in the form of various bulk properties of infinite nuclear matter-to better constrain the functional. Regardless, once the functional is properly defined and a fitting protocol established, minimization of the quality function defines the model. Until recently, once the minimum was found, one proceeded to validate the model against observables not included in the quality function [6]. Lately, however, a few studies have been devoted to map the landscape around the $\chi^{2}$ minimum [7-9]. Among the wealth of information revealed by such detailed statistical studies is the degree of correlation among various observables. Moreover, one can also use such a covariance analysis to assess the robustness of the model. 
For example, one could ask whether certain linear combinations of model parameters remain poorly constrained by the choice of observables. We find this to be particularly true in the case of the isovector sector that is hindered by the unavailability of highly accurate data on neutron skins [8].

It is the main goal of the present contribution to explore the feasibility of supplementing ground-state observables with nuclear excitations in the calibration of the quality function of relativistic functionals. We are confident that such an approach will relax the need for pseudodata while providing better constraints on the determination of the model parameters. Specifically, we advocate supplementing the quality function with centroid energies of monopole resonances for nuclei with a wide range of neutron-proton asymmetries. By doing so, the quality function becomes highly sensitive to the incompressibility of neutron-rich matter-which itself depends on both the compression modulus of symmetric matter and the slope of the symmetry energy [10-12].

Perhaps the most serious impediment to the implementation of this program is numerical in nature. By itself, computing the distribution of monopole strength for a wide range of excitation energies for models with finite-range interactions is numerically intensive [13]. Thus, embedding such RPA calculation into a complex $\chi^{2}$-minimization routine becomes impractical even for today's most powerful computers. However, the computation of centroid energies relies on knowledge of just a few moments of the distribution. In particular, an estimate of the centroid energy may be obtained from knowledge of two moments: the energy weighted sum $\left(m_{1}\right)$ and the inverse energy weighted sum $\left(m_{-1}\right)$. Remarkably, for nonrelativistic density functionals it has since long been established that the fully self-consistent $m_{-1}$ moment may be calculated quite generally from the ground-state properties of a slightly modified (i.e., "constrained") density functional [14-18], a result often referred to as the "dielectric theorem". Similarly, the $m_{1}$ moment may also be obtained by computing the ground-state expectation value of a suitable operator $\left(\left\langle r^{2}\right\rangle\right.$ in the case of the isoscalar monopole mode) [19]. Indeed, the constrained approach has been widely used in the nonrelativistic mean-field theory to study the centroid energy of the giant monopole resonance on a variety of nuclei, see Refs. [17,20-27], and references quoted therein. In particular, in response to significant experimental advances that have allowed the measurement of the distribution of monopole strength in neutron-rich nuclei [28,29], constrained Skyrme calculations have been used to predict the GMR centroid energies along different isotopic chains [24-26]. Measurements of the distribution of monopole strength along isotopic chains are sensitive to the incompressibility coefficient of asymmetric matter, and thus to the poorly known density dependence of the nuclear symmetry energy [11]. Therefore, for the systematic numerical exploration required to elucidate the isovector sector of the EDF, the constrained approach dramatically simplifies the computational effort as compared with the numerically intensive demands of self-consistent RPA calculations.

In contrast to the nonrelativistic case, only a handful of constrained calculations have been reported in the context of relativistic mean-field theory. As far as we know, the first relativistic constrained Hartree calculations of giant monopole energies of finite nuclei were performed at the end of the 1980s using the linear Walecka model [30,31]. Some time later, constrained monopole energies using nonlinear RMF models were reported in Ref. [32]. More recently, constrained RMF calculations of the $m_{-1}$ moment of the monopole mode were carried out in Ref. [36]. It is also worth mentioning that a somewhat different constrained approach, the so-called generator coordinate method (GCM), has been applied in RMF studies of giant monopole resonances [33-35]. However, the available literature with quantitative analyses of the degree of agreement between relativistic constrained calculations and the corresponding RPA results is very scarce; we are aware of only the RMF constrained-vs-RPA study of the $m_{-1}$ moment of the GMR in ${ }^{208} \mathrm{~Pb}$ reported in Ref. [36]. Moreover, to our knowledge the constrained approach has not been systematically implemented in the context of relativistic meanfield theories with the goal of supplementing the calibration of nuclear functionals with collective excitations. Our work aims at filling this gap in the literature.

The reason for such an imbalance between the constrained relativistic and nonrelativistic frameworks may be fairly evident. On the one hand, the derivation of the energy weighted sum rule (EWSR) hinges on a nonrelativistic kinetic energy operator (i.e., quadratic in the momentum), and on the other hand, the derivation of the inverse energy weighted sum rule through the dielectric theorem has been established only for nonrelativistic Hamiltonians. Thus, whereas the sum-rule theorems have been proven analytically in the nonrelativistic case, to our knowledge general proofs do not exist in the relativistic RPA theory (except for a suggestion in Sec. 3 of Ref. [36] that the relativistic RPA $m_{-1}$ moment can be obtained from a constrained RMF calculation). Nevertheless, extending the constrained approach to the relativistic domain seems plausible by the fact that accurately calibrated nonrelativistic and relativistic energy density functionals provide similar distributions of isoscalar monopole strength. In particular, DFT makes no demands on whether the nuclear functional should be of a relativistic or nonrelativistic character; the precise form of the functional then becomes a matter of convenience. It may also be mentioned that in recent constrained RMF calculations performed in the Thomas-Fermi approximation a close analogy of the results with the classical sum-rule expressions was reported [37].

In the present paper we perform a numerical study to explore the quality of the constrained RMF predictions against detailed relativistic RPA calculations. Inspired by the appeal of the constrained approach in the nonrelativistic case-and the absence (to our knowledge) of detailed proofs of relativistic sum-rule theorems - we consider the present numerical validation of the constrained approach in the relativistic domain both interesting and important. We also note that correlating GMR energies to the bulk properties of the equation of state (EOS) is critical in our quest for imposing meaningful constraints on the nuclear EOS at and below saturation density. To be able to draw general conclusions on such correlations, they should be systematically investigated using a variety of nuclear energy density functionals, both nonrelativistic and relativistic. Indeed, constrained calculations of the GMR energy are being 
used for exactly those reasons [38]. It thus seems timely and necessary to establish the accuracy of the constrained RMF predictions of giant monopole energies.

The manuscript has been organized as follows. In Sec. II we review the necessary formalism required to compute the excitation energy from the complete distribution of isoscalar monopole strength. In addition, we describe how the $m_{-1}$ and $m_{1}$ moments are computed in the constrained approach. In Sec. III we compare results obtained from the constrained approach against those extracted from the full distribution of strength. We conclude in Sec. IV with a summary of our results and plans for the future.

\section{FORMALISM}

The starting point for the calculation of the nuclear response is the interacting Lagrangian density of Ref. [39] supplemented by an isoscalar-isovector term first introduced in Ref. [40]. That is,

$$
\begin{aligned}
\mathscr{L}_{\text {int }}= & \bar{\psi}\left[g_{\mathrm{s}} \phi-\left(g_{\mathrm{v}} V_{\mu}+\frac{g_{\rho}}{2} \boldsymbol{\tau} \cdot \mathbf{b}_{\mu}+\frac{e}{2}\left(1+\tau_{3}\right) A_{\mu}\right) \gamma^{\mu}\right] \psi \\
& -\frac{\kappa}{3 !}\left(g_{\mathrm{s}} \phi\right)^{3}-\frac{\lambda}{4 !}\left(g_{\mathrm{s}} \phi\right)^{4}+\frac{\zeta}{4 !} g_{\mathrm{v}}^{4}\left(V_{\mu} V^{\mu}\right)^{2} \\
& +\Lambda_{\mathrm{v}}\left(g_{\rho}^{2} \mathbf{b}_{\mu} \cdot \mathbf{b}^{\mu}\right)\left(g_{\mathrm{v}}^{2} V_{\nu} V^{v}\right) .
\end{aligned}
$$

The Lagrangian density includes an isodoublet nucleon field $(\psi)$ interacting via the exchange of two isoscalar mesons, a scalar $(\phi)$ and a vector $\left(V^{\mu}\right)$, one isovector meson $\left(b^{\mu}\right)$, and the photon $\left(A^{\mu}\right)$ [41,42]. In addition to meson-nucleon interactions, the Lagrangian density is supplemented by four nonlinear meson interactions with coupling constants denoted by $\kappa, \lambda, \zeta$, and $\Lambda_{\mathrm{v}}$. The first two terms ( $\kappa$ and $\left.\lambda\right)$ are responsible for a softening of the equation of state of symmetric nuclear matter at normal density that results in a significant reduction of the incompressibility coefficient relative to that of the original Walecka model $[41,43,44]$. Indeed, such a softening is demanded by the measured distribution of isoscalar monopole strength in medium to heavy nuclei $[13,21,28,45-49]$. Further, $\omega$-meson self-interactions, as described by the parameter $\zeta$, serve to soften the equation of state of symmetric nuclear matter at high densities and at present can only be meaningfully constrained by the limiting masses of neutron stars [50]. Finally, the parameter $\Lambda_{\mathrm{v}}$ induces isoscalar-isovector mixing and is responsible for modifying the poorly constrained density dependence of the symmetry energy [40,51]. Tuning this parameter has served to uncover correlations between the neutron skin of a heavy nucleus-such as ${ }^{208} \mathrm{~Pb}$ - and a host of both laboratory and astrophysical observables.

A consistent mean-field plus RPA (MF + RPA) approach to the nuclear response starts with the calculation of various ground-state properties. This procedure is implemented by solving self-consistently the appropriate set of mean-field (i.e., Kohn-Sham) equations generated by the Lagrangian density given above [41]. For the various meson fields one must solve Klein-Gordon equations with the appropriate baryon densities appearing as their source terms. These baryon densities are computed from the nucleon orbitals that are, in turn, obtained from solving the one-body Dirac equation in the presence of scalar and timelike vector potentials. This procedure must then be repeated until self-consistency is achieved. What emerges from such a self-consistent procedure is a set of singleparticle energies, a corresponding set of Dirac orbitals, scalar and timelike vector mean-field potentials, and ground-state densities. A detailed implementation of this procedure may be found in Ref. [52]. Having computed various ground-state properties one is now in a position to compute the linear response of the mean-field ground state to a variety of probes. In the present case we are interested in computing the isoscalar monopole response as probed, for example, in $\alpha$-scattering experiments $[28,45-48]$. Although the MF+RPA calculations carried out here follow closely the formalism developed in much greater detail in Ref. [13], some essential details are repeated here for completeness.

The distribution of isoscalar monopole strength may be extracted from the imaginary part of a suitable polarization tensor that we compute in a consistent relativistic RPA approximation [53,54]. That is,

$$
\begin{aligned}
S_{L}(q, \omega) & =\sum_{n}\left|\left\langle\Psi_{n}|\hat{\rho}(\mathbf{q})| \Psi_{0}\right\rangle\right|^{2} \delta\left(\omega-\omega_{n}\right) \\
& =-\frac{1}{\pi} \Im \Pi_{\mathrm{RPA}}^{00}(\mathbf{q}, \mathbf{q} ; \omega),
\end{aligned}
$$

where $\Psi_{0}$ is the nuclear ground state and $\Psi_{n}$ is an excited state with excitation energy $\omega_{n}=E_{n}-E_{0}$. To excite isoscalar monopole modes a simple transition operator of the following form may be used:

$$
\hat{\rho}(\mathbf{q})=\int d^{3} r \bar{\psi}(\mathbf{r}) e^{-i \mathbf{q} \cdot \mathbf{r}} \gamma^{0} \psi(\mathbf{r}) .
$$

Here $\hat{\rho}(\mathbf{q})$ is the Fourier transform of the isoscalar (baryon) density and $\gamma^{0}=\operatorname{diag}(1,1,-1,-1)$ is the zeroth (or timelike) component of the Dirac matrices. Such a transition operator is capable of exciting all natural-parity states, including the isoscalar monopole $(E 0)$ mode of interest to this work. In the particular case of an $E 0\left(J^{\pi}=0^{+}\right)$excitation, the effective transition operator reduces to the following simple form:

$$
\hat{\rho}_{E 0}(\mathbf{q})=\int d^{3} r \bar{\psi}(\mathbf{r}) j_{0}(q r) \gamma^{0} \psi(\mathbf{r}),
$$

where $j_{0}(x)=\sin (x) / x$ is the spherical Bessel function of order zero. Finally, in the long-wavelength limit, the distribution of isoscalar monopole strength $R(\omega ; E 0)$ may be directly extracted from the longitudinal response. That is,

$$
\begin{aligned}
R(\omega ; E 0) & =\sum_{n}\left|\left\langle\Psi_{n}\left|\hat{r}^{2}\right| \Psi_{0}\right\rangle\right|^{2} \delta\left(\omega-\omega_{n}\right) \\
& =\lim _{q \rightarrow 0}\left(\frac{36}{q^{4}}\right) S_{L}(q, \omega ; E 0) .
\end{aligned}
$$

Connecting the nuclear response to the polarization tensor is highly appealing as one can bring to bear the full power of the many-body formalism into the calculation of observables that can be directly extracted from experiment [53,54]. Moreover, the spectral content of the polarization tensor is both illuminating and physically intuitive. However, enforcing the self-consistency of the formalism, while essential, is highly 
nontrivial. Yet maintaining self-consistency-in particular by using a residual particle-hole interaction that is identical to the one used to generate the mean-field ground state-is essential for the preservation of important symmetries and the decoupling of various spurious modes $[13,55]$. Finally, given the critical role that certain moments of the distribution of strength play in our discussion, we close this section with a few essential definitions and relations.

In general, the moments of the distribution of isoscalarmonopole strength are defined as follows:

$$
m_{n}(E 0) \equiv \int_{0}^{\infty} \omega^{n} R(\omega ; E 0) d \omega .
$$

In particular, the EWSR for the isoscalar monopole mode is given, for a nonrelativistic Hamiltonian, by [19]

$$
\begin{aligned}
m_{1}(E 0) & =\int_{0}^{\infty} \omega R(\omega ; E 0) d \omega \\
& =\frac{2 \hbar^{2}}{M} A\left\langle r^{2}\right\rangle \equiv \frac{2 \hbar^{2}}{M} \int r^{2} \rho(\mathbf{r}) d^{3} r,
\end{aligned}
$$

where $M$ is the nucleon mass and $\rho(\mathbf{r})$ is the ground-state baryon density normalized to the baryon number $A$. In essence, the power of the sum rule is that it relates a moment of the full RPA distribution to the mean-square value of the ground-state density. We note that the above classical sum rule is only valid in the absence of exchange and momentum-dependent forces [19]. Such forces modify the classical sum rules and their impact is traditionally accounted for by multiplying the right-hand side of Eq. (7) by a correction factor. Perhaps the best known case for the need of such a correction factor $(\kappa)$ is in the context of the photoabsorption cross section and the model-independent Thomas-Reiche-Kuhn sum rule (with $\kappa_{\mathrm{TRK}} \approx 0.2$ ) [19]. As mentioned earlier, the classical sum rules were derived using a nonrelativistic formalism so one may also need to correct for relativistic effects [56,57]. We assume here that such relativistic effects may also be subsumed into such a correction factor. However, to our knowledge, a fully relativistic counterpart to Eq. (7) does not exist in the relativistic RPA theory.

In addition to the EWSR a moment of critical importance to the present work is the inverse EWSR. In the nonrelativistic formalism, it has long been established that such a moment can also be computed from the ground-state density of the constrained system by invoking the "dielectric theorem" $[16,17]$. That is,

$$
\begin{aligned}
m_{-1}(E 0) & =\int_{0}^{\infty} \omega^{-1} R(\omega ; E 0) d \omega=-\frac{1}{2}\left[\frac{d}{d \lambda} A\left\langle r^{2}\right\rangle_{\lambda}\right]_{\lambda=0} \\
& =-\frac{1}{2}\left[\frac{d}{d \lambda} \int r^{2} \rho(\mathbf{r} ; \lambda) d^{3} r\right]_{\lambda=0} .
\end{aligned}
$$

The ground-state density in this case must be obtained by supplementing the mean-field potential with a "constrained" one-body term of the form $\lambda r^{2}$. The addition of such a term shifts the weight of the single-particle orbitals to the interior, thereby leading to a more compact system. The inverse EWSR measures the (negative) slope of the mean-square radius at $\lambda=$ 0 . The same constrained procedure can be easily implemented in the relativistic approach by adding such a harmonic onebody potential to the repulsive vector interaction. However, in contrast to the simplicity of the prescription, establishing a formal proof of the dielectric theorem for a relativistic Hamiltonian is likely to be more challenging. Indeed, with due allowance made for the suggestion in Sec. 3 of Ref. [36] that the relativistic RPA $m_{-1}$ can be extracted from the constrained RMF ground state, we have not found in the literature a general proof of the validity-or lack thereof-of the dielectric theorem within the relativistic theory. Nevertheless, for our numerical exploration we will follow the nonrelativistic approach and compute the relativistic constrained energy from the $m_{1}$ and $m_{-1}$ moments as follows:

$$
E_{\mathrm{con}}=\sqrt{\frac{m_{1}}{m_{-1}}} .
$$

Note that we have reserved the term "centroid energy" to the more conventional ratio of $E_{\text {cen }}=m_{1} / m_{0}$. In what follows we use RMF models to investigate the agreement between the constrained energy obtained using the classical sum rules [Eqs. (7) and (8)] and from the corresponding integrals derived from the RPA distribution of monopole strength. We believe this to be the first RMF study of its kind, except perhaps for the study of the $m_{-1}$ moment in ${ }^{208} \mathrm{~Pb}$ presented in Ref. [36]. As it will be shown in the next section, we find that the relativistic constrained calculation agrees with the corresponding RPA value to better than $2 \%$, suggesting that for many systematic applications the constrained calculation may be of enormous utility. The result is of obvious practical interest because of the pervasive availability of computer codes for RMF spherical ground-state calculations as opposed to the scarcity of relativistic RPA codes. Moreover, self-consistent relativistic RPA calculations are numerically expensive and not free of technical subtleties; whereas they are close to be prohibitive for large-scale investigations, constrained RMF calculations are simple and very fast.

\section{RESULTS}

We start the section by displaying in Fig. 1 the distribution of isoscalar monopole strength for ${ }^{208} \mathrm{~Pb}$ using the accurately calibrated FSUGold ("FSU") parametrization [58]. Model parameters for this and the NL3 model are listed in Table I. Note that the left-hand figure displays the energy weighted monopole strength whereas the right-hand panel the inverse energy weighted strength. We should also mention that due to the nonspectral character of the RPA approach [13], the particle-escape width is computed exactly within the model. Given that the distribution of monopole strength is defined as the long-wavelength limit of the longitudinal response [see Eq. (5)], we display the strength function for two small values of the momentum transfer to ensure the convergence of our results. Finally, the two insets display the cumulative sums relative to their corresponding sum rules computed from the constrained RMF approach as indicated in Eqs. (7) and (8). The insets indicate that the RPA response accounts for about $90 \%$ of the corresponding sum rules. However, the constrained energies agree to better than $2 \%$, namely, $E_{\text {con }}(\mathrm{CRMF})=$ 

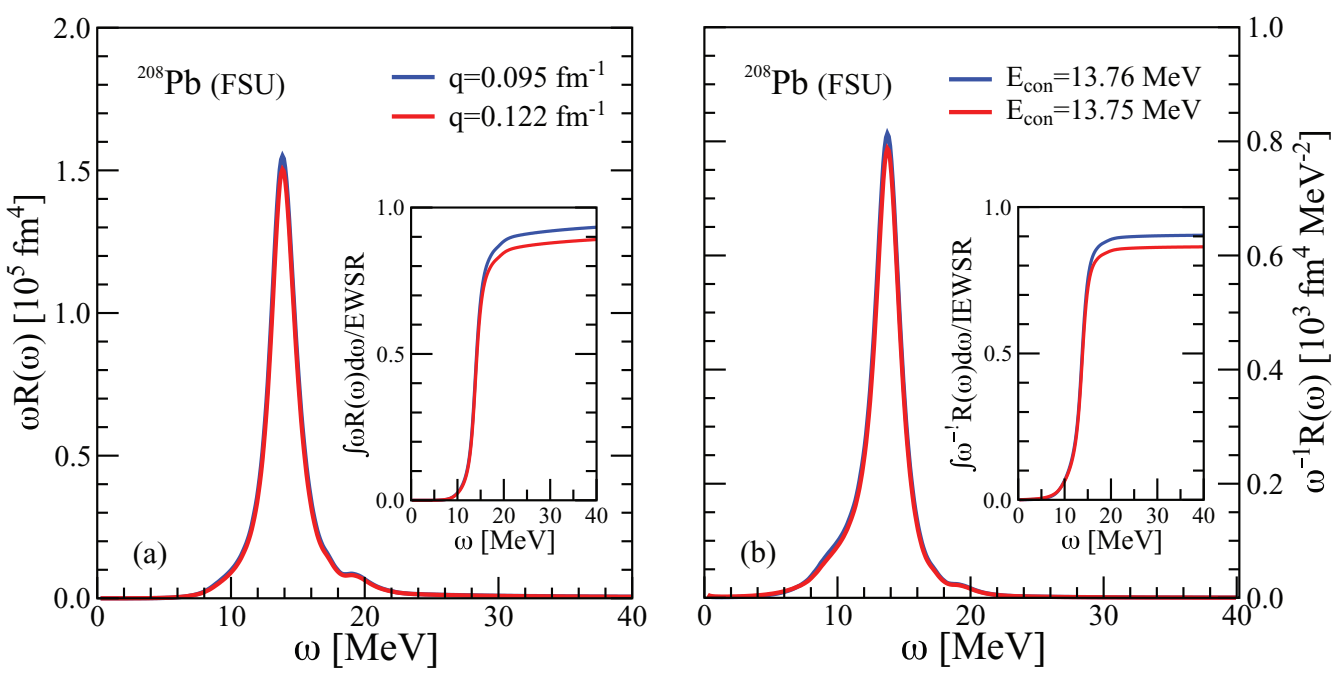

FIG. 1. (Color online) The energy-weighted monopole strength (a) and the inverse energy-weighted monopole strength (b) in ${ }^{208} \mathrm{~Pb}$ for two (small) values of the momentum transfer. The insets display the cumulative sums relative to the corresponding sum rules obtained from the constrained RMF approach [see Eqs. (7) and (8)].

$13.50 \mathrm{MeV}$ and $E_{\text {con }}(\mathrm{RPA})=13.76 \mathrm{MeV}$. Note, however, that this $2 \%$ difference represents the largest discrepancy obtained in the present work (see Table II).

Before proceeding any further with the discussion of our results we should mention some subtleties associated with the extraction of the constrained energy from the RPA results. Essentially, the potential problems emerge from the lack of convergence of the integrals over the excitation energy $\omega$ defining both $m_{1}$ and $m_{-1}$. Indeed, the lack of convergence of the energy-weighted and inverse energy-weighted sums can be clearly appreciated in the insets of Figs. 1(a) and 1(b), respectively. Note that this situation is not exclusive to the monopole resonance but extends to all excitation modes. To elucidate the problem we resort to an ideal Lorentzian distribution of strength that provides an accurate representation of the monopole strength in heavy nuclei, such as ${ }^{208} \mathrm{~Pb}$. The Lorentzian distribution is defined as follows:

$$
R(\omega)=m_{0} \frac{\Gamma / 2 \pi}{\left(\omega-\omega_{0}\right)^{2}+\Gamma^{2} / 4},
$$

where $m_{0}$ is the unweighted-energy sum and $\Gamma$ is the full width at half-maximum. Note that the full unweighted-energy sum $m_{0}$ can only be recovered by extending the integral over the unphysical $\omega<0$ region. That is,

$$
\int_{-\infty}^{\infty} R(\omega) d \omega=m_{0}
$$

However, by limiting the integral to the physical $\omega>0$ region, one may still account for most of the unweighted-energy sum-especially in the case that $\Gamma \ll \omega_{0}$. Indeed,

$$
\begin{aligned}
\int_{0}^{\infty} R(\omega) d \omega & =m_{0}\left[\frac{1}{2}+\frac{1}{\pi} \arctan \left(\frac{\omega_{0}}{\Gamma / 2}\right)\right] \\
& =m_{0}\left[1-\frac{1}{\pi}\left(\frac{\Gamma / 2}{\omega_{0}}\right)+\cdots\right] .
\end{aligned}
$$

In the particular case of ${ }^{208} \mathrm{~Pb}$ with the parameters predicted by the FSUGold parametrization $\left(\omega_{0}=13.82 \mathrm{MeV}\right.$ and $\Gamma=$ $2.29 \mathrm{MeV}$ ) one can still account for about $97 \%$ of the unweighted-energy sum. However, the situation is radically different with the $m_{1}$ and $m_{-1}$ moments as both integrals diverge; $m_{1}$ displays an ultraviolet divergence whereas $m_{-1}$ an infrared divergence-both logarithmic. Yet both integrals are well behaved if the integration region is allowed to be extended to the unphysical region. That is,

$$
\begin{aligned}
m_{1} & \equiv \int_{-\infty}^{\infty} \omega R(\omega) d \omega=m_{0} \omega_{0}, \\
m_{-1} & \equiv \int_{-\infty}^{\infty} \omega^{-1} R(\omega) d \omega=\frac{m_{0} \omega_{0}}{\omega_{0}^{2}+\Gamma^{2} / 4} .
\end{aligned}
$$

Note that in the case of an RPA distribution of an exact Lorentzian shape the centroid and constrained energies will

TABLE I. Parameter sets for the two accurately calibrated relativistic mean-field models used in the text: NL3 [59,60] and FSUGold [58]. The parameter $\kappa$ and the meson masses $m_{\mathrm{s}}, m_{\mathrm{v}}$, and $m_{\rho}$ are all given in $\mathrm{MeV}$. The nucleon mass has been fixed at $M=939 \mathrm{MeV}$ in both models.

\begin{tabular}{lcccccccccc}
\hline \hline Model & $m_{\mathrm{s}}$ & $m_{\mathrm{v}}$ & $m_{\rho}$ & $g_{\mathrm{s}}^{2}$ & $g_{\mathrm{v}}^{2}$ & $g_{\rho}^{2}$ & $\kappa$ & $\lambda$ & $\zeta$ & $\Lambda_{\mathrm{v}}$ \\
\hline NL3 & 508.194 & 782.501 & 763.000 & 104.3871 & 165.5854 & 79.6000 & 3.8599 & -0.015905 & 0.00 & 0.000 \\
FSU & 491.500 & 782.500 & 763.000 & 112.1996 & 204.5469 & 138.4701 & 1.4203 & +0.023762 & 0.06 & 0.030 \\
\hline \hline
\end{tabular}


TABLE II. Giant monopole resonance constrained and centroid energies for a variety of nuclei as predicted by the NL3 parametrization [59, 60]. Experimental data extracted from Refs. [45,61,62]. All quantities are given in MeV.

\begin{tabular}{lcccccc}
\hline \hline Nucleus & $\omega_{\min }-\omega_{\max }$ & $E_{\text {con }}(\mathrm{CRMF})$ & $E_{\text {con }}(\mathrm{RPA})$ & $\Delta E_{\text {con }}(\%)$ & $\omega_{\min }-\omega_{\max }$ & $E_{\text {cen }}(\mathrm{RPA})$ \\
\hline${ }^{16} \mathrm{O}$ & $0-50$ & 23.34 & 23.35 & 0.04 & $11-40$ & 23.95 \\
${ }^{40} \mathrm{Ca}$ & $0-50$ & 21.55 & 21.57 & 0.09 & $10-55$ & 21.95 \\
${ }^{90} \mathrm{Zr}$ & $0-40$ & 18.58 & 18.55 & 0.16 & $10-26$ & 18.54 \\
${ }^{116} \mathrm{Sn}$ & $0-40$ & 16.98 & 17.06 & 0.47 & $10-23$ & 17.03 \\
${ }^{144} \mathrm{Sm}$ & $0-40$ & 16.08 & 16.16 & 0.50 & $10-22$ & $17.89 \pm 0.37$ \\
${ }^{208} \mathrm{~Pb}$ & $0-40$ & 14.07 & 14.10 & 0.21 & $8-21$ & 16.10 \\
\hline \hline
\end{tabular}

be given by the following simple expressions:

$$
E_{\text {cen }}(\mathrm{RPA})=\omega_{0} \text { and } E_{\text {con }}(\mathrm{RPA})=\sqrt{\omega_{0}^{2}+\Gamma^{2} / 4} .
$$

So how does one extract the constrained energy from a distribution of strength that is physically meaningful only for positive values of $\omega$ ? Given the previous discussion, we suggest to rely heavily on the unweighted distribution of strength as it is both well behaved and accounts for almost $100 \%$ of the sum rule (at least in the Lorentzian approximation). Thus, we select the upper limit of integration $\left(\omega_{\max }\right)$ in such a way that most of the integrated strength $m_{0}$ has been accounted for. For the case of the energy-weighted sum, the remaining contribution (from $\omega_{\max }$ to $\infty$ ) is assumed to be exactly canceled by the contribution in the unphysical region. Finally, to remove the $\omega=0$ singularity in $m_{-1}$, we replace the low-energy tail by a rapidly falling exponential distribution in the interval $0<\omega<\omega_{\min }$. Given that the divergence at both low and high excitation energy is logarithmic in nature, we found our results stable against small changes in both $\omega_{\min }$ and $\omega_{\max }$.

Having explained how we extract RPA centroid and constrained energies, we now continue with the discussion of our results. Although the use of mean-field methods for light nuclei may be questionable-especially since the distribution of monopole strength may be strongly fragmented-we have performed self-consistent RPA calculations for nuclei ranging from ${ }^{16} \mathrm{O}$ to ${ }^{208} \mathrm{~Pb}$ using the NL3 and FSUGold energy density functionals. Considering these two functionals is useful because, although they both provide accurate descriptions of a variety of nuclear ground-state properties, their predictions for infinite nuclear matter are significantly different [11]. In particular, the incompressibility coefficient of symmetric nuclear matter predicted by NL3 is about $271 \mathrm{MeV}$ whereas that of FSUGold is $230 \mathrm{MeV}$. We provide our results in both tabular form in Tables II and III, and graphical form in Fig. 2. First, we note that the NL3 results for the centroid energies are systematically higher than the corresponding FSUGold results, as expected given the higher incompressibility coefficient of the NL3 interaction. The NL3 centroid energies also are systematically higher than the experimental data except in the case of ${ }^{208} \mathrm{~Pb}$. The case of ${ }^{208} \mathrm{~Pb}$ is unique because the large incompressibility of NL3 is compensated by a similarly large slope of the symmetry energy; this accounts for the accurate prediction of the centroid energy in ${ }^{208} \mathrm{~Pb}$ - but not in ${ }^{90} \mathrm{Zr}$ [10]. In essence, the FSUGold interaction was conceived with the goal of better constraining the incompressibility of neutron-rich matter by incorporating information on giant resonances into the calibration procedure. However, in the present work we aim to go one step further by exploring the feasibility of incorporating monopole energies directly into the calibration of the quality function. The results presented in both of the tables are extremely encouraging as they suggest differences of at most $2 \%$ between energies computed with the constrained RMF approach and those extracted from the RPA strength function. For reasons that at present we do not understand, the discrepancy between constrained and RPA results is systematically higher in the case of the FSUGold parametrization. Nevertheless, the accuracy of our results gives us confidence that in the future, constrained GMR energies for nuclei ranging from ${ }^{90} \mathrm{Zr}$ to ${ }^{208} \mathrm{~Pb}$ - and including a variety of $\mathrm{Sn}$-isotopes [12,28,63]—-may be directly included in the accurate calibration of energy density functionals. Such a procedure will not only be able to better constrain the density dependence of the equation of state around saturation density, but may be helpful in partly removing the reliance on "pseudodata" (i.e., on bulk properties of infinite nuclear matter).

TABLE III. Giant monopole resonance constrained and centroid energies for a variety of nuclei as predicted by the FSUGold parametrization [58]. Experimental data extracted from Refs. [45,61,62]. All quantities are given in MeV.

\begin{tabular}{lcccccc}
\hline \hline Nucleus & $\omega_{\min }-\omega_{\max }$ & $E_{\text {con }}(\mathrm{CRMF})$ & $E_{\text {con }}(\mathrm{RPA})$ & $\Delta E_{\text {con }}(\%)$ & $\omega_{\min }-\omega_{\max }$ & $E_{\text {cen }}(\mathrm{RPA})$ \\
\hline${ }^{16} \mathrm{O}$ & $0-50$ & 22.89 & 23.09 & 0.87 & $11-40$ & 23.68 \\
${ }^{40} \mathrm{Ca}$ & $0-50$ & 20.67 & 20.66 & 0.05 & $10-55$ & 21.18 \\
${ }^{90} \mathrm{Zr}$ & $0-40$ & 17.70 & 17.94 & 1.34 & $10-26$ & 17.88 \\
${ }^{116} \mathrm{Sn}$ & $0-40$ & 16.20 & 16.48 & 1.70 & $10-23$ & $16.18 \pm 0.37$ \\
${ }^{144} \mathrm{Sm}$ & $0-40$ & 15.37 & 15.52 & 0.97 & $10-22$ & $17.89 \pm 0.20$ \\
${ }^{208} \mathrm{~Pb}$ & $0-40$ & 13.50 & 13.76 & 1.89 & $8-21$ & 13.81 \\
\hline \hline
\end{tabular}




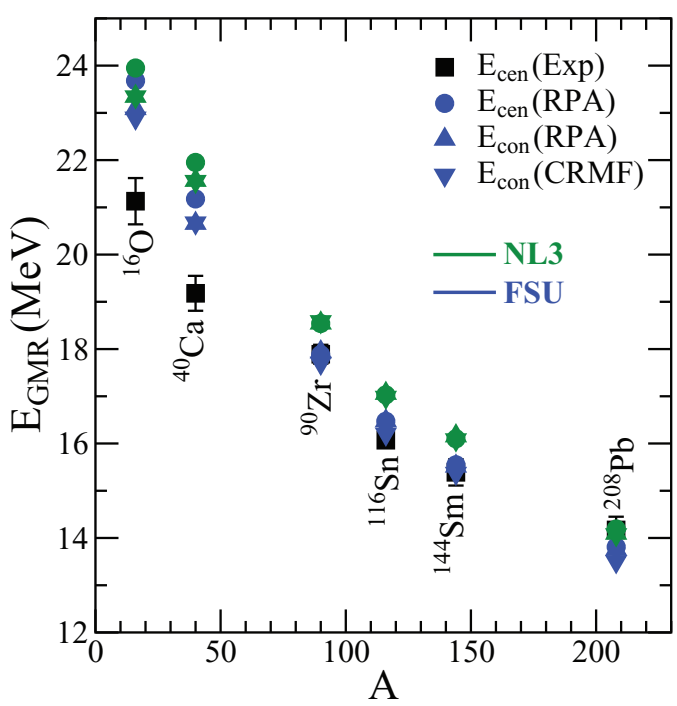

FIG. 2. (Color online) Centroid and constrained energies for a variety of nuclei as predicted by the NL3 $[59,60]$ and FSUGold [58] models. Experimental centroid energies are taken from Refs. [45,61,62].

\section{CONCLUSIONS}

The sum-rule approach provides a powerful tool for the analysis of nuclear excitation spectra [19]. Particularly useful are the energy-weighted sum rules-with the TRK sum rule as its best known exponent-as they can be determined largely model-independently from a few well-known ground-state properties. Moreover, additional sum rules, such as the inverse energy-weighted sum rule, may be combined to estimate the mean excitation energy of the resonances from intrinsic ground-state properties. However, the standard analytical proofs of the validity of the sum-rule theorems rely on a nonrelativistic Hamiltonian. Such is the case of the modelindependent EWSR based on commutation relations, and of the inverse EWSR extracted from constrained calculations of the ground state. Thus, the sum-rule approach to giant monopole energies has been limited to the nonrelativistic domain; for a recent and successful implementation of such techniques see Ref. [20], and references therein. Indeed, in Ref. [20] highly accurate Skyrme-based mean-field plus RPA calculations are successfully compared against constrained predictions for the mean excitation energy of various resonances.

In this contribution we have conjectured that the constrained approach to the isoscalar GMR may be extended to the relativistic domain. We were prompted by the fact that in different applications, as long as the energy density functional is accurately calibrated to physical data, the specific form of the nuclear functional becomes a matter of convenience. Indeed, accurately calibrated nonrelativistic and relativistic energy density functionals provide a similar distribution of the isoscalar monopole strength. We believe that assessing the degree of agreement in the relativistic domain between the predictions of the constrained approach and the RPA results is reason enough to implement the present study. However, our study also has an important practical component. Given that we aim to eventually supplement the calibration of energy density functionals with experimental data on nuclear collective modes, the validity of the constrained approach becomes essential, as it is impractical to incorporate this information from a detailed RPA calculation.

Relativistic mean-field plus RPA calculations were performed for the distribution of isoscalar monopole strength for magic or semimagic nuclei ranging from ${ }^{16} \mathrm{O}$ to ${ }^{208} \mathrm{~Pb}$ using two accurately calibrated density functionals (NL3 and FSUGold). Predictions were made for both centroid and constrained energies from the various moments of the distribution. RPA calculations of this kind are computationally expensive as the strength distribution is finely mapped over a wide energy range. In contrast, the extraction of monopole energies from the constrained approach is numerically expedient as it relies exclusively on the self-consistent evaluation of ground-state properties. As previously conjectured, we find excellent agreement between the two approaches. Indeed, for all nuclei under consideration the discrepancy between the two approaches amounts to less than $2 \%$, a quantity that is comparable to current experimental errors.

In summary, relativistic constrained calculations of monopole energies were favorably compared against the corresponding predictions from the relativistic RPA approach. These results are highly encouraging and show the promise of incorporating nuclear excitations in the calibration of future energy density functionals without incurring in an unaffordable computational cost.

\section{ACKNOWLEDGMENTS}

M.C. is grateful to Prof. J. Martorell for useful discussions. This work was supported in part by the United States Department of Energy under Grant No. DE-FG05-92ER40750. M.C. acknowledges support from the Consolider Ingenio 2010 Programme CPAN CSD2007-00042, from Grant No. FIS2011-24154 from MICINN and FEDER, and from Grant No. 2009SGR-1289 from Generalitat de Catalunya.
[1] Building a universal nuclear energy density functional, (UNEDF Collaboration), http://unedf.org.

[2] M. Kortelainen, T. Lesinski, J. More, W. Nazarewicz, J. Sarich et al., Phys. Rev. C 82, 024313 (2010).

[3] P. Hohenberg and W. Kohn, Phys. Rev. 136, B864 (1964).

[4] W. Kohn and L. J. Sham, Phys. Rev. 140, A1133 (1965).

[5] W. Kohn, Rev. Mod. Phys. 71, 1253 (1999).
[6] J. Piekarewicz, Phys. Rev. C 76, 064310 (2007).

[7] P.-G. Reinhard and W. Nazarewicz, Phys. Rev. C 81, 051303(R) (2010).

[8] F. J. Fattoyev and J. Piekarewicz, Phys. Rev. C 84, 064302 (2011).

[9] F. J. Fattoyev and J. Piekarewicz, Phys. Rev. C 86, 015802 (2012).

[10] J. Piekarewicz, Phys. Rev. C 69, 041301(R) (2004). 
[11] J. Piekarewicz and M. Centelles, Phys. Rev. C 79, 054311 (2009).

[12] J. Piekarewicz, J. Phys. G 37, 064038 (2010).

[13] J. Piekarewicz, Phys. Rev. C 64, 024307 (2001).

[14] D. J. Thouless, Nucl. Phys. 21, 225 (1960).

[15] D. J. Thouless, Nucl. Phys. 22, 78 (1961).

[16] E. R. Marshalek and J. da Providência, Phys. Rev. C 7, 2281 (1973).

[17] O. Bohigas, A. Lane, and J. Martorell, Phys. Rep. 51, 267 (1979).

[18] P. Ring and P. Schuck, The Nuclear Many-Body Problem (Springer, New York, 2004).

[19] M. N. Harakeh and A. van der Woude, Giant ResonancesFundamental High-frequency Modes of Nuclear Excitation (Clarendon, Oxford, 2001).

[20] T. Sil, S. Shlomo, B. K. Agrawal, and P.-G. Reinhard, Phys. Rev. C 73, 034316 (2006).

[21] G. Colò, N. V. Giai, J. Meyer, K. Bennaceur, and P. Bonche, Phys. Rev. C 70, 024307 (2004).

[22] P. Gleissl, M. Brack, J. Meyer, and P. Quentin, Ann. Phys. (NY) 197, 205 (1990).

[23] J. P. Blaizot, J. F. Berger, J. Dechargé, and M. Girod, Nucl. Phys. A 591, 435 (1995).

[24] M. Centelles, X. Viñas, S. K. Patra, J. N. De, and T. Sil, Phys. Rev. C 72, 014304 (2005).

[25] L. Capelli, G. Colò, and J. Li, Phys. Rev. C 79, 054329 (2009).

[26] E. Khan, Phys. Rev. C 80, 011307(R) (2009).

[27] E. Khan, J. Margueron, G. Colò, K. Hagino, and H. Sagawa, Phys. Rev. C 82, 024322 (2010).

[28] T. Li et al., Phys. Rev. Lett. 99, 162503 (2007).

[29] D. Patel et al., Phys. Lett. B 718, 447 (2012).

[30] T. Maruyama and T. Suzuki, Phys. Lett. B 219, 43 (1989).

[31] H. F. Boersma, R. Malfliet, and O. Scholten, Phys. Lett. B 269, 1 (1991).

[32] M. Stoitsov, M. Cescato, P. Ring, and M. Sharma, J. Phys. G 20, L149 (1994).

[33] M. V. Stoitsov, P. Ring, and M. M. Sharma, Phys. Rev. C 50, 1445 (1994).

[34] D. Vretenar, G. Lalazissis, R. Behnsch, W. Poschl, and P. Ring, Nucl. Phys. A 621, 853 (1997).

[35] M. Sharma, Nucl. Phys. A 816, 65 (2009).

[36] Z. Y. Ma, N. V. Giai, A. Wandelt, D. Vretenar, and P. Ring, Nucl. Phys. A 686, 173 (2001).
[37] M. Centelles, S. K. Patra, X. Roca-Maza, B. K. Sharma, P. D. Stevenson, and X. Viñas, J. Phys. G 37, 075107 (2010).

[38] E. Khan, J. Margueron, and I. Vidaña, Phys. Rev. Lett. 109, 092501 (2012).

[39] H. Mueller and B. D. Serot, Nucl. Phys. A 606, 508 (1996).

[40] C. J. Horowitz and J. Piekarewicz, Phys. Rev. Lett. 86, 5647 (2001).

[41] B. D. Serot and J. D. Walecka, Adv. Nucl. Phys. 16, 1 (1986).

[42] B. D. Serot and J. D. Walecka, Int. J. Mod. Phys. E 6, 515 (1997).

[43] J. D. Walecka, Ann. Phys. (NY) 83, 491 (1974).

[44] J. Boguta and A. R. Bodmer, Nucl. Phys. A 292, 413 (1977).

[45] D. H. Youngblood, H. L. Clark, and Y.-W. Lui, Phys. Rev. Lett. 82, 691 (1999)

[46] M. Uchida et al., Phys. Lett. B 557, 12 (2003).

[47] M. Uchida, H. Sakaguchi, M. Itoh, M. Yosoi, T. Kawabata et al., Phys. Rev. C 69, 051301(R) (2004).

[48] T. Li, U. Garg, Y. Liu, R. Marks, B. Nayak et al., Phys. Rev. C 81, 034309 (2010).

[49] J. Piekarewicz, Phys. Rev. C 66, 034305 (2002).

[50] P. Demorest, T. Pennucci, S. Ransom, M. Roberts, and J. Hessels, Nature 467, 1081 (2010).

[51] C. J. Horowitz and J. Piekarewicz, Phys. Rev. C 64, 062802 (2001).

[52] B. G. Todd and J. Piekarewicz, Phys. Rev. C 67, 044317 (2003).

[53] A. L. Fetter and J. D. Walecka, Quantum Theory of Many Particle Systems (McGraw-Hill, New York, 1971).

[54] W. H. Dickhoff and D. Van Neck, Many-body Theory Exposed (World Scientific Publishing Co., Singapore, 2005).

[55] J. F. Dawson and R. J. Furnstahl, Phys. Rev. C 42, 2009 (1990).

[56] S. M. Cohen, Adv. Quantum Chem. 46, 241 (2004).

[57] H. Sinky and P. T. Leung, Phys. Rev. A 74, 034703 (2006).

[58] B. G. Todd-Rutel and J. Piekarewicz, Phys. Rev. Lett 95, 122501 (2005).

[59] G. A. Lalazissis, J. Konig, and P. Ring, Phys. Rev. C 55, 540 (1997).

[60] G. A. Lalazissis, S. Raman, and P. Ring, At. Data Nucl. Data Tables 71, 1 (1999).

[61] D. H. Youngblood, Y.-W. Lui, and H. L. Clark, Phys. Rev. C 63, 067301 (2001).

[62] Y.-W. Lui, H. L. Clark, and D. H. Youngblood, Phys. Rev. C 64, 064308 (2001).

[63] J. Piekarewicz, Phys. Rev. C 76, 031301(R) (2007). 